\title{
OBSERVATIONAL TESTS IN COSMOLOGY
}

\author{
James E. Gunn
}

Hale Observatories, California Institute of Technology, Carnegie Institution of Washington

Nous testons les trois paramètres sans dimension décrivant les modèles cosmologiques de Friedmann : le paramètre de décélération $q_{0}$, la densité $\Omega_{0}$, et le paramètre temps $\tau_{0}=\mathrm{H}_{0} \mathrm{t}_{\mathrm{o}}$. N'importe quel choix de deux de ces paramètres, compte-tenu de l'échelle donnée par la constante de Hubble, décrit un modèle d'univers dans le cadre de la relativité générale avec une constante cosmologique non nulle. L'état actuel des observations et des développements théoriques sont passés en revue. Quelques hypothèses sont proposées. Seuls les tests pour $\Omega$ o sont valables à l'heure actuelle toutefois des doutes subsistent. Les données et l'état de compréhension actuels suggèrent fortement que $\Omega_{\mathrm{o}}$ est petit, probablement inférieur à 0.1 . Par contre, les tests pour $q_{0}$ et $\tau_{0}$ requièrent tous deux de meilleures observations et un gros travail théorique avant de pouvoir espérer des réponses définitives.

\section{INTRODUCTION}

Perhaps the most profound realization of modern cosmology is that the universe is a dynamical entity. Prodded first by the discovery of the expansion, and then by the theoretical setting offered by Einstein's General Theory, we have come to understand that the central questions about the structure of the universe in the large are dynamical ones, and that the size, shape, and qualitative temporal behavior of the universe are determined by its dynamics. In this presentation I shall try to outline the current role of optical observations in addressing dynamical 
questions in cosmology. The primary optical tool, of course, is the redshift of distant objects, but recent work has indicated that as much or more is to be learned by understanding well the structures relatively nearby as by use of the extremely difficult 'global' cosmological tests, though there are some questions that can only be answered by those tests.

of paramount importance, and probably the most difficult question to answer, is "which theory?" We shall see that the observations are so limited by fundamental statistical and technological difficulties that at most a few parameters can be determined. History is replete with theories and notions that have primarily a cosmological motivation, and that are either impotent or incorrect when applied to laboratory and solarsystem dynamical experiments. It seems prudent to avoid them, and to adopt as one's working framework a theory which is logically complete and has passed the tests so far asked of it; most would agree that General Relativity and possibly some variants of it [e.g. scalar-tensor gravity d la Brans and Dicke (1961)] are the most promising candidates. We consider only General Relativity here, and admit as the only possible heresy the inclusion of the Cosmological Constant, though there is as of this writing no real evidence that it does not vanish. If one further admits that the cosmic microwave background is in fact "cosmic" and is a relic of the singular origin of the universe, one's choice of models is narrowed for all practical purposes (for epochs later than a redshift of several thousand, anyhow) to the Friedmann isotropic, homogeneous models, and it is to these we restrict our attention. These models are determined by three parameters, two dimensionless and one with dimensions of length or time which does nothing but set the scale. The dimensional one is conventionally taken to be the Hubble time $\left(\mathrm{H}_{\mathrm{O}}^{-1}\right)$. The dimensionless ones are most conveniently taken to be either

$$
\Omega_{0}=\frac{8 \pi G \bar{\rho}_{O}}{3 H_{O}^{2}}=\frac{\bar{\rho}_{O}}{\rho_{C}} \text {, and } q_{O}=-\frac{\ddot{R} R}{R^{2}}=\frac{\Omega_{0}}{2}-\frac{\Lambda}{3 H_{0}^{2}}
$$


or

$$
\tau_{0}=H_{0} t_{0} \text { and } \Omega_{0} \text { or } q_{0} \text {, }
$$

depending on the tests under consideration. Here $\bar{\rho}_{0}$ is the present mean density of matter, $t_{0}$ the age of the universe, and $\Lambda$ the cosmological constant. A concise summary of the variety of models as described by these parameters is given in figure 1 , taken from Gunn and Tinsley (1975). The inset boxes show the schematic time development of the Friedmann scale factor $R$, to which all distances in the expanding frame are proportional. The diagram is cut by several curves which separate grossly different characteristics. Models to the right and below the " $k=0$ " line have the curvature index $k=-1$, and are (under the simplest topological assumptions) open and infinite. Models along the $k=0$ line are spatially flat (and open and infinite), while those above and to the left of the curve are closed and

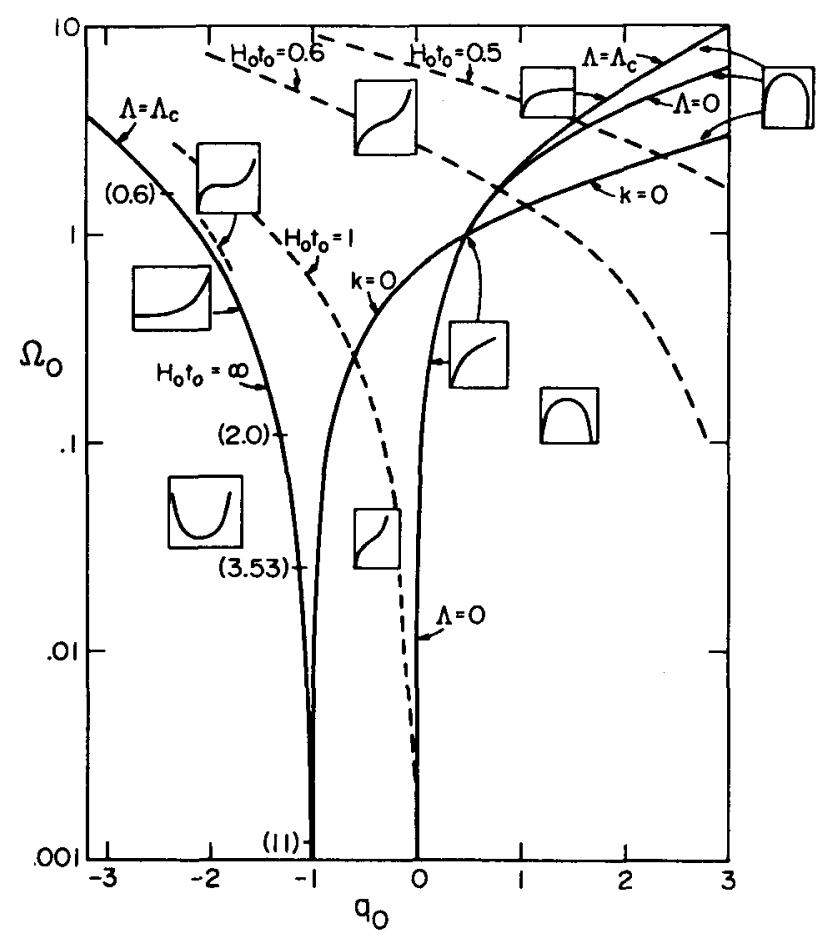

Fig. 1: The $q_{0}{ }^{-\Omega}$ o diagram for General Relativistic Cosmological models with nonzero $\Lambda$. The inset boxes illustrate the qualitative behavior of $R(t)$ for various models. See text for fuller description. 
finite, with $k=+1$. Models below and to the right of the $\Lambda=0$ curve have negative $\Lambda$, corresponding to an attractive harmonicoscillator cosmological force; such models always expand to a maximum and collapse again (the "potential barrier" of the $\Lambda$ force is infinite in this case). Models above and to the left of the $\Lambda=0$ line have positive $\Lambda$ (repulsive $\Lambda$-force) and exhibit a great variety of behavior. The right branch of the $\Lambda=\Lambda_{c}$ curve (the one which joins the $\Lambda=0$ curve) separates models which expand indefinitely into the future from those which reach a maximum and collapse. Along the $\Lambda=0$ curve, of course, that separation is at $k=0$; positively curved closed models collapse and negatively curved open ones expand forever. Models to the left of the left branch of the $\Lambda=\Lambda_{C}$ curve have no Big Bang, and can probably be ruled out (Gunn and Tinsley 1975). Thus independent knowledge of any pair of the parameters $q_{0}$ ' $\tau_{0}$ ' or $\Omega_{0}$ is sufficient to uniquely specify the geometry and the entire time development of the model to within a scale, which is provided by $\mathrm{H}_{0}$. The nature of the experiments used to determine the parameters is dictated by the nature of the parameters themselves, and can be conveniently divided into the general classes "local" and "global," depending on whether distances very small compared to, or of the order of, $1 / \mathrm{H}_{0}$ must be surveyed. The tests for $q_{0}$ are all global and exceedingly difficult; those for $\Omega_{0}$ and $\tau_{0}$ involve easier observations, but the interpretation is often far from straightforward.

We shall not discuss the Hubble constant here, since it has been dealt with elsewhere in these proceedings. It is fortunate that knowledge of it is important for our purposes only for the determination of $\tau_{0}$, in which it enters directly. $q_{0}$ is scale-independent, as are, fortunately, most of the evolutionary corrections thereto, and for $\Omega_{o^{\prime}}$, the quantity of interest is $\bar{\rho}_{\mathrm{o}} / \mathrm{H}_{\mathrm{O}}{ }^{2}$, which for virially-derived masses is also independent of $\mathrm{H}_{\mathrm{O}}$.

II. THE DETERMINATION OF $\tau_{0}$

Let us discuss first the determination of $\tau_{0} \cdot$ If one knows the Hubble constant, one needs to know the age of the uni- 
verse. Let us suppose for the moment that the cosmological constant vanishes. Then the dividing line between open, indefinitely expanding models and closed, cataclysmic ones occurs at $\tau_{0}=2 / 3$, and the relevant question is whether the observed values are significantly smaller than, or larger than, that value. With $\mathrm{H}_{\mathrm{O}}=50$, the critical age is about 13 billion years. Current estimates from nuclear chronometers span that figure, with newer estimates based on the Rhenium-Osmium chronometer giving somewhat larger ages than before. The range based on "plausible" models now seems to be about 11-18 billion years (Hainebach and Scl.samm 1976, Gott, Gunn, Schramm, and Tinsley 1974, hereinafter GGST), but the dependence on the very uncertain models for galactic evolution is quite severe (Tinsley 1975). To the age of the elements must be added the time of formation of the Galaxy, which probably does not exceed $10^{9}$ years but is very uncertain. Added to this, of course, is the uncertainty in the Hubble constant itself, understanding of which awaits at the very least some understanding of the anisotropies which have long been discussed by de Vaucouleurs and at this conference by Dr. Rubin. Another possible source for the determination of $\tau_{0}$, of course, are the ages of the oldest stars, specifically the ages of globular cluster stars. Here the chief uncertainties are uncertainties in the theory of stellar evolution and uncertainties in the helium abundance. Helium abundances can be obtained from RR Lyrae pulsation characteristics in comparison with models thereof, but there is no real evidence that the relatively large values of $\mathrm{Y}$ obtained do not reflect some mixing from hydrogenburning regions in the interior, and indeed there seems to be ample evidence accumulating that mixing does play some role in Population II giant evolution. [See, for example, the recent work by Auer and Demarque (1976).] One consistent approach is to use the helium abundance predicted by Big-bang nucleosynthesis, and to determine the globular cluster ages using that helium abundance; since the Big-bang value of $Y$ depends itself on $\tau_{0}$ through the current density, one should be able to obtain a self-consistent value for $\tau_{0}$, if one can persuade oneself that $\mathrm{H}_{\mathrm{O}}$ is well enough known. This is sadly not the case, as 
is illustrated in figure 2, where it is seen that the slope of the helium abundance with age for the Big-bang production is very nearly the same as the inferred helium abundance with age for the globular-cluster models of Iben (1967). The test therefore gives essentially no information on $\tau_{0}$ ' but does set some constraints on $\mathrm{H}_{0}$ if one is willing to trust the models that far. We return to this point later, but for now it is sufficient to remark that for $\Omega_{0}$ 's between 0.5 and 0.02 , which seems a plausible range, values for $\mathrm{H}_{0}$ between 50 and 60 seem reasonable. Thus current techniques for determination of $\tau_{0}$ do not yield very helpful results, but it is heartening to note that the range of values is plausible, which one hardly needs to be reminded was not always the case. It would appear that much better understanding of galactic chemical evolution and/or much better theoretical understanding of Population II stellar evolution must be in hand before the currently available methods for obtaining $\tau_{0}$ will yield useful answers.

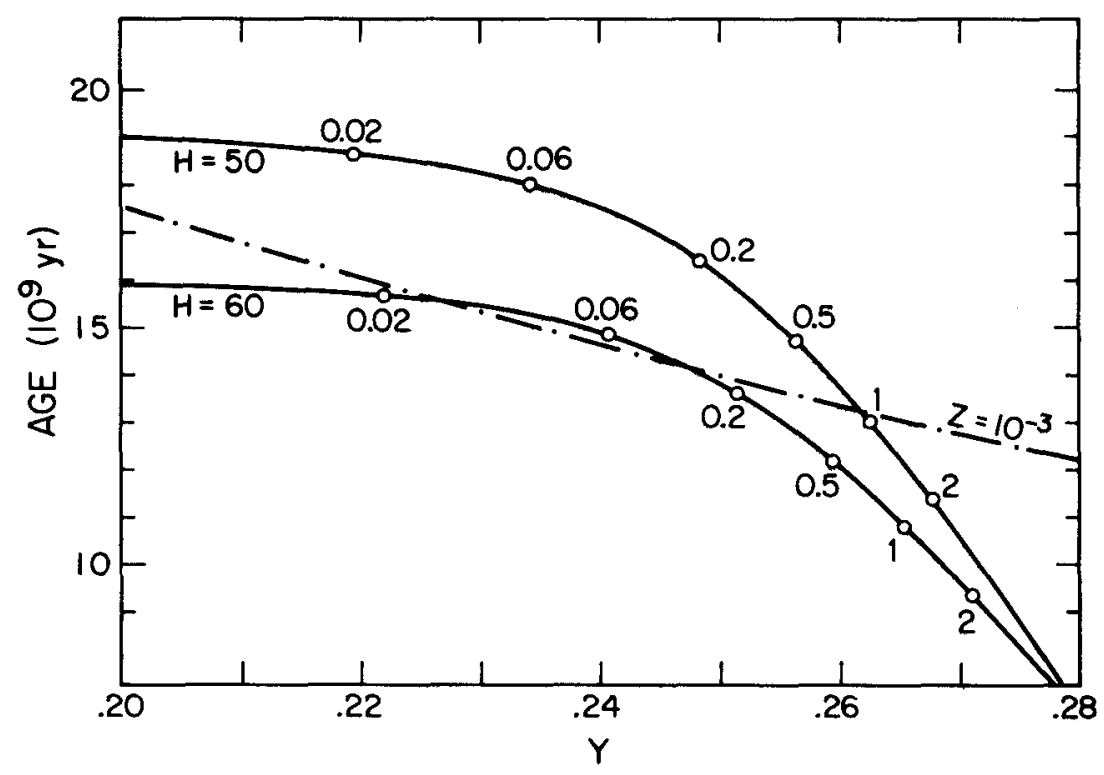

Fig. 2. Comparison of the ages obtained as a function of helium abundance for $\Lambda=0$ models from primordial synthesis (curved lines) and from globular cluster stars (nearly straight line) for 2 values of the Hubble constant. The numbers along the curved lines are values of $\Omega_{0}$. 
III. TESTS TO DETERMINE $q_{0^{\circ}}$

Let us now turn our attention to tests for the determination of $q_{0}$; as remarked earlier, these tests are all global, since they must sample enough of the run of $R(t)$ to get information on its second derivative. The classical method of determining $q_{0}$ is by means of the Hubble diagram; the angulardiameter-redshift test is theoretically equivalent and in practice manifests some of the same problems; we shall discuss them both.

In principle, both tests are simplicity itself; one measures in the one case the flux from a set of sources of known intrinsic luminosity and redshift, and in the other the angular diameter of a set of sources of known linear size and redshift, and compares with the predictions of models. To first order, as we shall see, the only dependence is on $q_{0}$; other effects enter through higher derivatives on $R$ and through the curvature, and these appear first as terms quadratic in the redshift.

In practice, the difficulties are so far overwhelming; there are four main categories of trouble that afflict both tests:

1. The objects being photometered or measured do not have edges; thus "aperture corrections" to the measurements exist.

2. There exist distance-dependent selection effects large enough to give completely fraudulent results unless extreme care is exercised.

3. The objects are not quite standard; thus the test is essentially statistical, and one must get enough data to achieve statistical accuracy and use estimators that are not biased. The difficulty of obtaining the data demands that very efficient estimators be used, but it is often difficult to estimate the bias in such estimators.

4. The objects evolve with time in both brightness and size, and one must understand that evolution to very high accuracy in order to make corrections of the requisite precision. 
The requirement that the objects be reasonably standard and that evolutionary corrections be at least in principle obtainable dictates thus far that the objects used for the Hubble diagram test be galaxies, the favored class to date being the brightest ellipticals in clusters of galaxies. These objects exhibit a small dispersion in brightness, less than 0.4 magnitudes [Humason, Mayall, and Sandage (1956)], and most their light is contributed by a stellar population that was surely roughly coeval with the galaxy. Thus their evolution is not seriously complicated by unknown current rates of star formation, which would make the calculation of evolutionary corrections for giant spirals, for example, hopelessly difficult. The difficulties are large enough for ellipticals, as we shall see.

The monochromatic flux $F$, received from an isotropically emitting source at redshift $z$, is related to the luminosity $L$ by the relation

$$
F_{\nu /(1+z)}=\frac{L_{\nu} H_{0}^{2}}{4 \pi z^{2}(q, \Omega, z)(1+z) c^{2}}
$$

in which all the cosmological information is contained in the function $Z(q, \Omega, z$ ) [cf. Gunn and Oke (1974), hereinafter GO]. This relation can be written to first order in the redshift as

$$
m_{v /(1+z)}=-2.5 \log L_{v}+f(z)-\frac{2.5}{\ln 10} q_{0} z+0\left(z^{2}\right)
$$

where $\mathrm{m}$ in the monochromatic magnitude and $f(z)$ is a function which does not depend on the cosmology. Thus to first order the only cosmological dependence is on $q_{0}$. To quite acceptable accuracy the aperture corrections for photometry can be written

$$
L_{v}=L_{v}^{0}\left(r / r_{0}\right)^{\alpha}
$$

where $r_{0}$ is some fiducial radius and $I_{0}$ the luminosity within that projected radius, and $\alpha$ is about 0.7 at $r_{0}=15 \mathrm{kpc}$ for giant ellipticals. This dependence of luminosity on aperture 
size lowers the sensitivity of the Hubble diagram test to $q_{0}$, since for large $q_{0}$, the objects appear brighter and larger, so an aperture of given size admits less light, canceling some of the increase in brightness. Including this effect in expression (4) and also including the effects of evolution, we have

$m_{v /(1+z)}=-2.5 \log L_{\nu, t_{0}}^{o}+g(z)-\frac{2.5}{\ln 10} \frac{2-\alpha}{2} z\left[q_{0}-\frac{2}{2-\alpha} \frac{1}{\tau_{0}} \frac{d \ln L}{d \ln t}\right]+0\left(z^{2}\right)$

where again only terms to first order in the redshift have been retained, and $g(z)$ is again a function which does not depend on the cosmological model. One sees immediately that the effects of evolution enter the expression in the same way as do changes in $\mathrm{q}_{\mathrm{O}^{\prime}}$ and hence that errors in the evolution rates are in this order indistinguishable from such changes.

Inspection of relation (6) shows that an error in magnitude $\Delta \mathrm{m}$, either because of selection effects, improper correction for aperture effects, or miscalculated evolutionary corrections, will incur an error $\Delta \mathrm{q}_{0} \cong 1.4 \Delta \mathrm{m} / \mathrm{z}$. The currently available surveys have most of their weight at $z^{\prime} s$ around 0.3 , so to obtain $q_{0}$ to an accuracy of 0.5 requires that the mean magnitude at that redshift be determined to 0.1 ; one would like to do much better, since the difference for $\Lambda=0$ between an empty and a just-closed model is that big, and to obtain an accuracy of 0.1 , say, with a sample at $z=0.5$ requires knowing the mean magnitude to within 0.035. Leaving aside for the moment the problem of the evolutionary corrections, let us consider the statistical problem. The first thing to notice is that the required accuracy for interesting results is much smaller than the intrinsic dispersion of brightness in the sample galaxies, so that extraordinary care must be exercised to avoid bias.

There are very many known possible sources of bias, and probably as many which have not yet surfaced. It is probably worth while here to discuss a few of these briefly. It is imperative, for example, not to make "corrections" which cannot be applied with equal accuracy to both nearby and distant objects; correcting the light for faint companions which may fall in the 
diaphragm, but which by virtue of loss of resolution or faintness cannot even be seen at large redshifts, is an obvious example. There is a large class of "Scott effect" type sources of bias, some of which have been investigated and some not. The classical case (Scott 1957) is, of course, that at large distances the easiest clusters to find are very rich ones, and rich clusters should have brighter brightest members than poor clusters. This effect has been investigated by Sandage (see, for example, Sandage 1976), who concludes that the effect is not as large as one might expect if the brightest cluster galaxies are drawn from the general luminosity function. The effect can be calibrated, but the difficulty is that the calibration must be done accurately, and rich clusters are so rare that they must be found at large distances where cosmological effects are substantial. That requiras that one must have less rich clusters at those distances to make comparisons, and it may reasonably be asked why the less rich ones are not used to do the test in the first place.

A less obvious effect occurs with the use of radio galaxies for the Hubble diagram at large redshifts; the appeal for their use is the same as that for using the bright members of rich clusters, namely that they are easy to find. The dangers in their use have become obvious only recently, with the work of Colla et al. (1975) and of Jaffe and Perola (1976). These workers have found an effect which should have at least been suspected, namely that there is a strong correlation of optical brightness with radio brightness. Colla et al. find that the mean absolute magnitude $\left\langle\mathrm{M}_{\mathrm{v}}\right\rangle$ is related to the $408 \mathrm{MHz}$ radio absolute magnitude $M_{R}$ by

$$
<M_{v}>=0.2 M_{R}+\text { const }
$$

up to $\log \mathrm{P}_{408}$ of 25.6. At the limit of the $3 \mathrm{CR}$, this corresponds to a redshift of about 0.07 ; the corresponding optical magnitude is very near the mean of the brightest cluster galaxies. At redshifts of 0.4 to 0.7 where current work is being done on the redshifts of radio galaxies, the radio power levels 
are very much higher, and the space densities of such objects is extremely low. Since the radio luminosity function is so steep, in any given catalog one finds the bulk of the objects near the lower flux limit of the catalog, which means that one must look at more and more powerful sources as one goes to larger and larger redshifts. The situation is illustrated in figure 3 . The extrapolated colla et al. relation results in an error in $q_{0}$ of almost 3 for a sample of radio galaxies alone with redshifts of about 0.5 . The luminosity function for galaxies becomes very much steeper at about this brightness. One might thus expect a change of slope, and there is indeed evidence that the relation flattens, but the statistics are of necessity rather poor. Calibrating the effect seems hopeless again without a sample of non-radio galaxies at comparable redshifts which is large enough already to perform the test for $q_{0}$ quite satisfactorily.

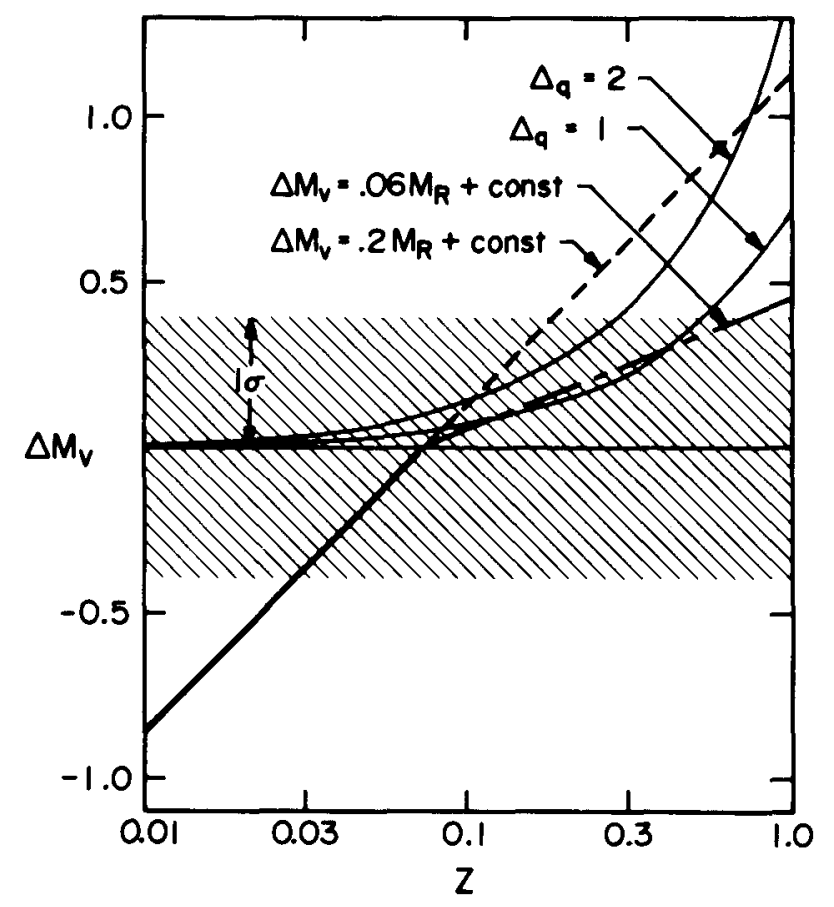

Fig. 3. The relation between $z$ for a source at the lower flux limit of the $3 C R$ and mean visual magnitude, according to Colla et al., shown extrapolated as is, and with a reduction of slope by a factor 3 beyond the level they analyze. Curves of equivalent change in $q_{0}$ are plotted as well; the cross-hatched region is the $\pm 1 \sigma$ area for non-evolving brightest cluster galaxies. 
All this is not to say that very rich clusters and distant radio galaxies are not interesting objects in their own right, but their usefulness for the Hubble diagram test is dubious at best, and completely misleading at worst.

It thus seems imperative to use a sample which is chosen in as nearly uniform a manner as possible, with care taken to choose the same type of cluster at all distances, with the same criteria for choosing the brightest member and measuring it; any corrections must be of a kind that can be applied uniformly at all distances. It is also important to look for the clusters on plate material which is graded in depth and in wavelength, so that for clusters in a given distance range, one looks near the same rest wavelength (for large redshifts, spirals, since they are bluer, become brighter than ellipticals in a given fixed optical band; the character of the clusters and the brightest cluster galaxies will thus change in a systematic way as one goes to larger and larger distances) and at about the same level above the plate limit. Oke and the author (GO) have attempted to do this; the large-redshift part of their survey is not yet complete, but some preliminary results from an intermediate-redshift sample have been published in the above reference; these results will be commented on later.

Another source of bias comes from the treatment of the data themselves. The sample of clusters is not complete except at small redshifts. This relative deficiency of sample points at large redshifts causes a familiar Malmquist effect, here complicated by the k-corrections, the redshift effect on flux, and the complicated way in which the volume grows with redshift (which, of course, is itself dependent on the cosmological model). These effects are discussed in GO, and a solution is offered there, namely to treat the sampling magnitude, i.e.,the magnitude system in which the clusters are found, as the independent variable and find the distribution of redshift with that apparent magnitude for a given model, and fit that to the data. That scheme is fine as long as the appearance in the sample or not of a cluster is determined by the brightness of its brightest member, and that in turn is $O K$ as long as one is limited by 
the faintness to which one can get redshifts, but has a sample which goes much fainter. This is paradoxically no longer the case; with modern detectors one can obtain redshifts for essen. tially any cluster one can find, and the whole question of selection must be carefully rethought for the faint sample now almost in hand. The problem offers no easy solution.

The data for the intermediate $G O$ sample is illustrated in figure 4, along with several theoretical curves for the mean, uncorrected for the Malmquist effect. The abscissa $V_{I}$ is the monochromatic magnitude of a wavelength corresponding to $5456 \AA$ in the rest frame of the galaxy. Two curves of constant $V$ apparent magnitude are also given, illustrating the necessity of transforming the data to the sampling magnitude for statistical analysis.

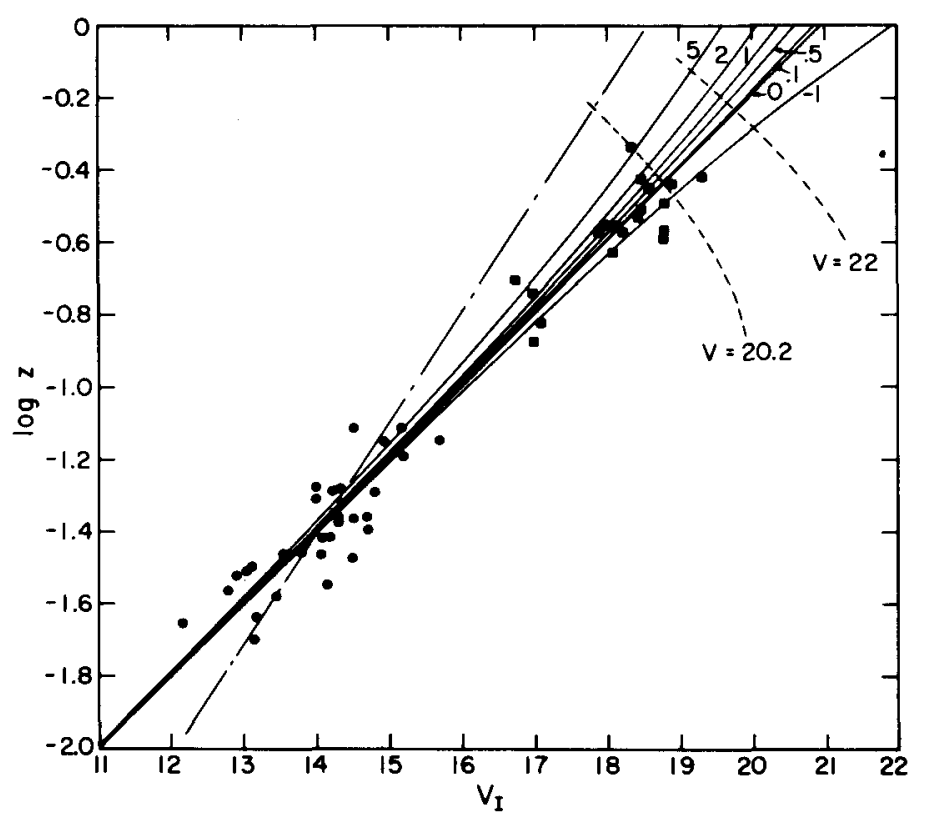

Fig. 4. The Hubble diagram from the Gunn-Oke data with curves (not including the Malmquist correction) for several values of $q_{0}$. The dot-dashed line is the prediction of the model of Segal (1972).

The last question of a statistical nature one needs to consider is how large the sample needs to be in order to achieve a given accuracy. From equation (6), one easily finds an approximate relation for the standard deviation of the formal 
value of $q_{0}$ :

$$
\sigma_{q_{0}}=1.4\left(\sigma_{\mathrm{mL}}^{2}+\sigma_{\mathrm{mH}}^{2}\right)^{1 / 2}\left(\left\langle\mathrm{z}_{\mathrm{H}}>-\left\langle\mathrm{z}_{\mathrm{L}}>\right)^{-1}\right.\right.
$$

Here we have arbitrarily divided the sample into a highredshift part with mean redshift $\left\langle\mathbf{z}_{\mathrm{H}}\right\rangle$, and a low-redshift part with mean redshift $\left\langle\mathbf{z}_{\mathrm{L}}\right\rangle, \sigma_{\mathrm{mL}}$ and $\sigma_{\mathrm{mH}}$ are the standard deviations in the magnitudes of the two samples from the model relation of best fit, and for a model which fits are evidently just $\sigma / \mathrm{N}_{\mathrm{L}} 1 / 2$ and $\sigma / \mathrm{N}_{\mathrm{H}}{ }^{1 / 2}$, where $\sigma$ is the dispersion in the absolute magnitude of the sample galaxies. There are about 100 Abell clusters of richness 1 or greater at galactic latitudes greater than 30 degrees and redshifts less than about 0.1 ; 40 or so more can be expected to be found on the southern hemisphere survey. Thuan and the author have undertaken to measure the redshifts and obtain photometry for this sample, which seems to be analogous to the one being found in the Go survey at large redshifts. The mean redshift is about 0.05 , and if $\sigma$ is 0.4 , the contribution to the error in $q_{0}$ is 0.12 for a high redshift sample most of whose weight is at a redshift of about 0.5 , which seems a reasonable limit for the present. Thus a sample of 100 clusters at that redshift would contribute an error of like size, for a total error of about 0.17 ; obtaining a smaller error involves more work than is likely to be feasible for some time to come. The error is uncomfortably large, since $q_{0}=0$ and $q_{0}=1 / 2$ are separated by only about $3 \sigma$, but it would seem that that is the best one is likely to do.

In order to make use of that accuracy, however, one must know the evolutionary correction to better than 0.04 magnitudes at $z=0.5$, or, expressed another way, one must know din $L / d$ in $t$ to about 0.1 . Dr. Tinsley will discuss the problem of the evolutionary corrections elsewhere in the symposium in some detail; we will consider here only the main known sources of the corrections and the order-of-magnitude estimates made to date. One correction, of course, comes from the fact that the stars of which the galaxies are made age and die, and new giants (which 
contribute most of the light at all optical wavelengths) evolve from lower-and-lower mass main sequence stars. With the best parameters one can determine today (Tinsley and Gunn 1976a), the contribution to dlnL/dlnt is about -1.0 , but that is uncertain by at least 0.3 and perhaps by more. The corresponding change in $q_{0}$ is between 1.5 and 2 , depending on the cosmological model, in the sense that the measured value in the absence of the correction is too large (galaxies at large redshifts are too bright). This is offset (probably) by an effect pointed out by Ostriker and Tremaine (1975); the large central galaxies in clusters tend to accrete smaller galaxies by the process of dynamical friction. The phenomenon has also been investigated by simon White at Cambridge, and will be discussed by him at this symposium. Tinsley and the author (Gunn and Tinsley 1976) have attempted to assess the observational consequences of the effect, with the conclusion that it is quite impossible at this time to be sure even what the sign of the effect is, much less how large it is, but the order of magnitude is likely to be similar to the stellar evolution term, corresponding to an uncertainty in $q_{0}$ of one or two. It is difficult not to be pessimistic, but the problem is clearly not impossibly difficult, and there is much effort being expended by many workers attempting to understand the effect. The application of the Hubble diagram in any meaningful way clearly awaits better understanding of the evolutionary corrections than we have in hand at the moment.

The results to date from the Hubble diagram technique are not very accurate formally, because of the small samples and small redshift range, but are already sufficiently precise that the largest uncertainty is evolution. Go obtain a value of $-0.15 \pm 0.6$ (sd) using the intermediate-redshift sample discussed above. When these data are analyzed using the Thuan-Gunn Abell low-redshift sample for the bright end, this value falls by about 0.1 and the standard error is reduced somewhat. Sandage, Kristian, and Westphal (1976a,b) have unpublished results which extend to a larger redshift for which no formal analysis has been done, but they surmise that the data fit $q_{0}=1$ reasonably well. The high-redshift part of the sample is, however, domi- 
nated by radio sources, so the interpretation will be quite difficult.

A variant of the Hubble diagram test as described makes use not of the brightness of the first-ranked cluster galaxies but the brightness of some standard place in the normalized luminosity function for the whole cluster [Bautz and Abell (1973), schechter (1976), Peach and Austin (1975)]. It is possible that such tests have more formal power than the brightest-galaxy test, in the sense that the dispersion of the standard luminosity is smaller, but understanding the evolutionary effects presents an appalling problem, much more difficult, in the author's opinion, than the corresponding one for the brightest galaxy. It may be, however, that in order to understand the dynamical effects in sufficient detail to be able to calculate corrections for the brightest galaxy, one must understand the whole Iuminosityfunction evolution. If this is so, and if there is any success, the luminosity-function test is essentially independent (statistically) of the brightest-galaxy test, and, like the clusterdiameter test to be described below, can be used to complement the ordinary Hubble-diagram test to yield better accuracy. If all three tests can be used and the formal accuracies are as good as the preliminary work indicates, a sample of the sort discussed for the Hubble diagram can yield formal errors for $q_{0}$ of order 0.08 . Needless to say, the evolution will also have to be understood to twice the precision discussed if that accuracy is to be utilized.

The Hubble-diagram test is, in all geometric theories of gravity, equivalent to the angular-diameter test from a theoretical point of view [that is to say, they determine the same quantity, namely $z(q, \Omega, z)$ and therefore to first order only $\left.q_{0}\right]$, and indeed we have seen that the introduction of the aperture correction already involves cosmological effects on angular diameters in the Hubble diagram. Petrosian (1976) has recently pointed out that, by observing the quantity $I(r) / \bar{I}(r)$, where $I$ and $\bar{I}$ are the surface brightness of a galaxy at radius $r$ and the mean surface brightness interior to $r$, respectively, one can define a scale and consequently a metric diameter 
for a galaxy. The metric diameter for galaxies is unfortunately subject to dynamical evolution just as the brightness is, and in a way which seems not to be simply related to the changes in brightness. The technique promises to be a valuable check on dynamical evolution models when they exist, however, and several efforts are being mounted to make the necessary observations. The situation regarding clusters of galaxies is somewhat different, and cluster diameters may turn out to be the most useful global test available. The core diameter of clusters, definable in a variety of ways, appears to be a stable statistic, probably of comparable power in determining $q_{0}$ to the brightness of the brightest cluster galaxies. Zwicky (1957) first pointed out this stability, and it has been investigated since by a number of authors, notably Bahcall (1973), and most recently and perhaps most exhaustively by Hickson (1976). Hickson uses a suitably defined spatial covariance function to define diameters, and though the technique suffers an "aperture correction" not unlike that for photometry, it is independent of the large-scale shape and such problems as centering. Background corrections are straightforward, and the statistics are easily modeled and understood. The evolutionary corrections are not entirely understood yet, but promise to be simpler than for galaxies, and almost certainly smaller. Figure 5 shows Hickson's data, which best fit a value of $q_{0}$ near -1 after crude evolutionary corrections are allowed for. The formal statistical error in this result is about 0.4 (one sigma). There is no evidence for correlations of diameter with richness or morphology, but the same caveats apply here as in the Hubble diagram case, and a homogeneous set of data must be a prerequisite to a trustworthy answer. The technique shows great promise, and has the added advantage that it is entirely independent of the Hubble diagram but can make use of the same redshift data, and so in principle at least doubles the usefulness of those very difficult-to-obtain data.

It would thus seem that the results of the available global tests are as yet not very decisive nor are they likely to become so very quickly. A vast amount of both theoretical and observa- 


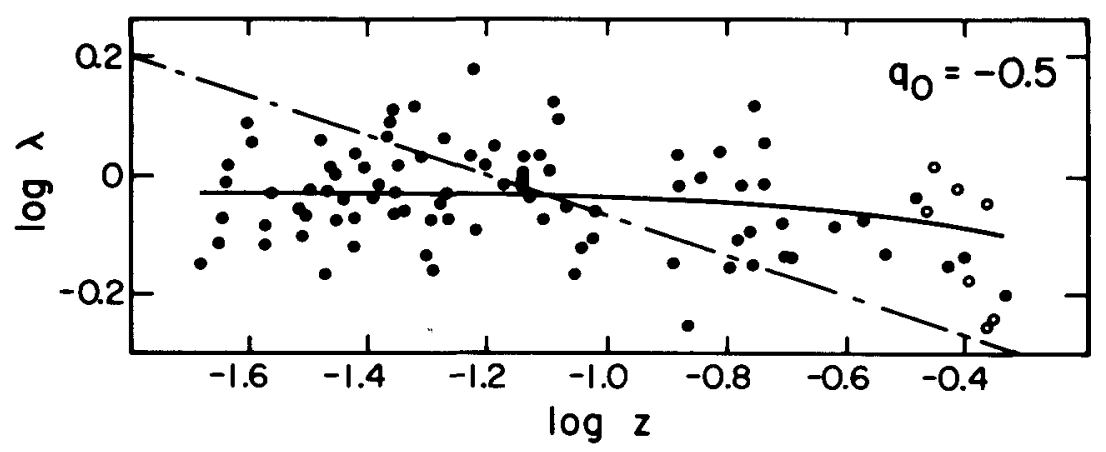

Fig. 5. The linear-diameter-redshift data of Hickson; the theoretical curve corresponding to $q_{0}=-0.5$ is superposed. The dotdashed line is the prediction of the theory of Segal (1972).

tional work will be required to make them so. The problem is sufficiently important, however, that one may be sure that the work will be done.

IV. TESTS TO DETERMINE $\Omega_{0}$.

The tests to be described here do not make use of data on very distant galaxies, but use redshift data on relatively nearby objects to formulate dynamical criteria for determining the local mean density. The situation has been reviewed extensively in GGST, so the treatment here will be restricted to recent developments and a sketchy outline of past results. The tests are basically of two types: in the first, one attempts to measure the luminosity density $\mathcal{L}$ in a suitably large region in our neighborhood, and then attempts to measure a representative mass-to-light ratio. The product of those quantities is then the required density. The two measurements depend on the Hubble constant in such a way, as remarked earlier, that a value of $\Omega_{0}$ emerges directly and is independent of the assumed value of $\mathrm{H}_{0}$. The most recent determination of the luminosity density is the one obtained by Gott and Turner (1976), which agrees well with past determinations which have corrected for the local enhancement caused by the local supercluster. They obtain a value of 
4. $7 \times 10^{7} \mathrm{~L}_{\odot} \mathrm{Mpc}^{-3}$. (For $\mathrm{H}_{0}=50 \mathrm{~km} \mathrm{~s}^{-1} \mathrm{Mpc} ;$ all M/L's are photographic and refer to the Zwicky catalogue photometric system.) The values of $M / L$ come from applications of the virial theorem to various structures. It has become clear that the $\mathrm{M} / \mathrm{L}$ 's of the visible bodies of galaxies are much too small to be representative of the mean (see, for example, Ostriker, Peebles, and Yahil 1974), and that the $M / L^{\prime}$ 's increase as one goes to larger scales. How far this trend continues is the major unanswered question, but there are compelling theoretical reasons to believe that it must stop at scales of about 1 Mpc (Gunn 1976) unless the density of the universe is dominated by some very hot, noninteracting fluid (see GGST for a discussion of this case).

Consideration of the dynamics of the local group and other small groups, in which most of the light and presumably most of the mass in the universe resides, yields $\mathrm{M} / \mathrm{L}$ 's of about 100 (Gunn 1974, Gott and Turner 1976, Geller and Peebles 1974). The masses of great clusters of galaxies are much larger than those of these small structures, and the photographic $M / L^{\prime}$ s are also somewhat greater, by a factor of 2 to 3 . This difference can easily be understood, however, on the basis of the difference in stellar population in the galaxies in small groups and in great clusters. The bulk of the light in great clusters comes from ellipticals and lenticulars with no young stellar population, whereas that from the small groups is dominated by spirals, most of whose light comes from relatively young stars. Stripping a spiral of gas and dust and waiting a few billion years produces a system with half to a quarter the photographic light, depending on the time dependence of star formation in the original spiral with which the stripped one is compared; thus one would expect, all else being equal, for the big clusters to exhibit mass-to-light ratios higher by roughly the observed amount. Thus there is no reason to believe that the great clusters exhibit a larger proportion of "hidden mass" than small groups, and it seems likely that the relevant $M / L$ is not too different from 100 , normalized to a typical spiral stellar population. (This ratio of about 3 in M/L for spirals as compared to ellipticals has been found independently by Turner (1976) from a study of binary 
galaxies.) This $\mathrm{M} / \mathrm{L}$ and the above-mentioned luminosity density yield an $\Omega_{0}$ of about 0.06 ; the main uncertainties are systematic and arise from the assumption that the mass is distributed more or less like the light. Nearly all current theories of galaxy formation suggest that that should be the case, but it is by no means certain that it is so.

The second class of tests for $\Omega_{0}$ involves the investigation of perturbations to the expansion caused by relatively small-amplitude, large-scale perturbations in density like the local supercluster. They are in actuality generalizations of the $M / L \times \mathcal{L}$ test discussed before, but are sufficiently different in application that we consider them separately. They are also in principle very much more powerful, since they sample very much larger regions of space, though the presence of a very hot, uniform fluid also invalidates them, and they are not independent of $\Lambda$ if $\Lambda$ is large (of order $4 \pi \mathrm{G} \rho / \mathrm{c}^{2}$ ).

Sandage, Tammann, and Hardy (1972) first pointed out that the perturbation in the local expansion rate brought about by the local supercluster is a sensitive function of $\Omega_{0}$. The theoretical situation has been discussed since by GGST, Silk (1974), and Peebles (1976a). The basic idea is that the effect of gravitation is much larger in the overall dynamics of dense universes, so the gravitational effects on the dynamics of an overdense perturbation are much larger than they are for a perturbation of the same relative amplitude in lower-density models. The situation is illustrated in figure 6 , in which the ratio of the Hubble constant inside a uniform perturbation of given density contrast to that far away from it is plotted for various contrasts and values of $\Omega_{0}$. The current observational situation is not at all clear; Sandage and Tammann (1976) claim to see no effect whatever, which at their level of precision suggests that $\Omega_{0}$ is less than about 0.1 . Peebles (1976), analyzing the same data, finds that the statistics are not good enough to conclude even that $\Omega_{0}$ is less than unity, though marginally better fits are obtained with small $\Omega_{0}$. De Vaucouleurs (1976), again analyzing the same data, suggests that large effects are present, and that a ratio of asymptotic to local Hubble constant of 
nearly 1.5 is suggested. He also finds rotation or shear which cannot be accounted for by the models which have been used to discuss the perturbations and which indeed suggest that there are serious troubles with the basic ideas underlying our understanding of the growth of perturbations in the universe. The same difficulties attend the observations of Rubin et al. (1976) which have been presented at this symposium, and until they are understood this author, at least, advises extreme caution in the interpretation of the local supercluster data.

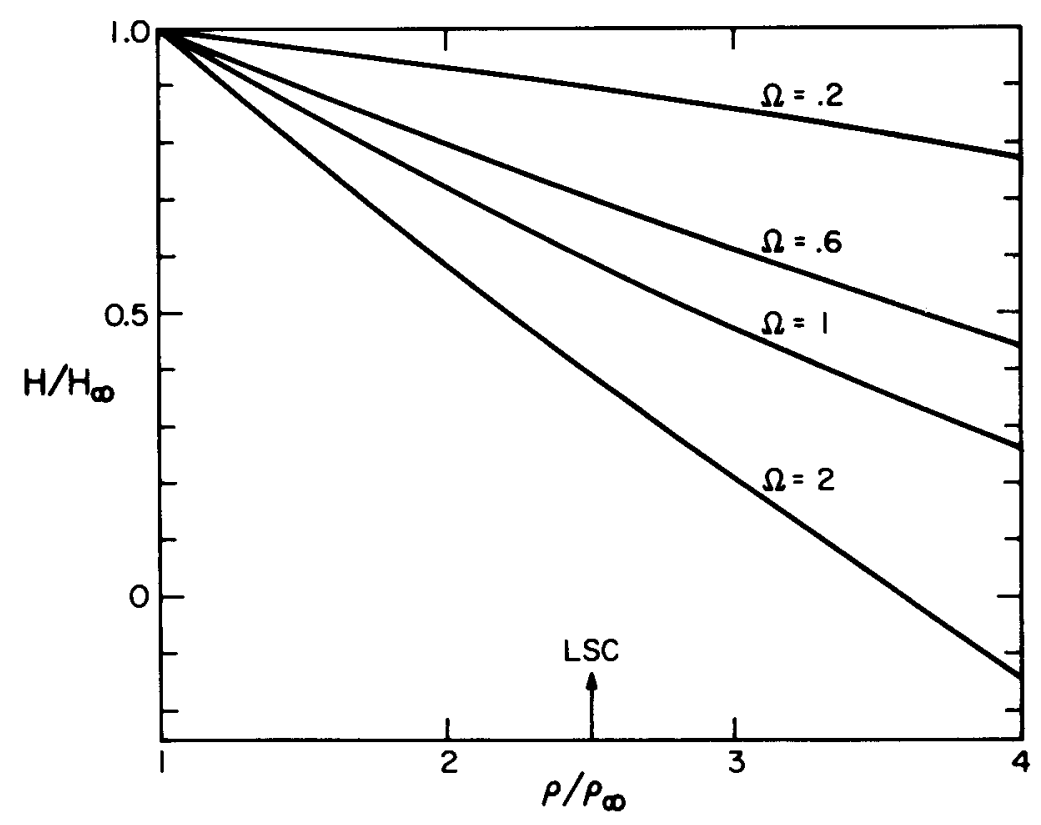

Fig. 6. The value of local to asymptotic Hubble constants as a function of the density contrast $\rho / \bar{\rho}$ for a uniform density perturbation for several values of $\Omega_{0} \quad(\Lambda=0)$.

There are several related tests which should be mentioned. Various formulations of the "Cosmic Virial Theorem" (Fall 1976, Peebles 1976b), which state that the kinetic energy of motions with respect to a uniformly expanding frame should be comparable with the potential energy of clustering, give tests for $\Omega_{0}$ ' since the kinetic energy scales like $\Omega_{0}$ and the potential like $\Omega_{0}{ }^{2}$. The tests again suggest that $\Omega_{0}$ is small, of order a few hundredths, but Peebles point out that the interpretation is not straightforward because the platform from which we observe 
is not quite like the one which the Virial Theorem analysis uses. Gott and the author (1976) have formulated a test based on the fact that the gravitational acceleration of the galaxy due to another galaxy is just proportional to its observed brightness, if the $M / L ' s$ of galaxies are sensibly the same. Comparing the predictions for the local velocity field with observations again suggests that $\Omega_{0}$ is small, probably less than 0.1 .

There is, however, some dynamical evidence that $\Omega_{0}$ is not so small. In his study of the projected covariance density for the distribution of galaxies on the sky, Peebles (1975 and references contained therein) finds that the covariance function is well representable by a single power law over all accessible scales. Theoretical arguments (Peebles 1974) indicate that this should not be so unless $\Omega_{0}$ is near unity. Gott and Rees (1975) argue, however, that if the initial perturbation spectrum is not white noise (in the sense that there is more fluctuation power at large scales than given by white noise, which is in fact supported by other evidence), the observations can be understood in the context of models with small $\Omega_{0} \cdot \mathrm{N}$-body simulations to test these ideas are in progress both by Gott and Aarseth and by Peebles and Groth; there are, however, some difficulties in interpreting the results due to boundary and finite-N effects.

V. SUMMARY

We have reviewed the available data for the determination of the three dimensionless cosmological parameters, $q_{0}{ }^{\prime} \tau_{0}$, and $\Omega_{0}$, any two of which suffice in principle to determine the structure and time development of the universe up to a scale which is provided by the Hubble constant, assuming that the correct theory of gravity is General Relativity with a cosmological constant. Much of the repugnance with which $\Lambda$ has been regarded is due to its ugliness when it is regarded as part of the field equations, that is, part of the left-hand side of the field equations. A more modern viewpoint, perhaps, is that it might arise from an imperfect cancellation of the gravitational effect of quantum vacuum fluctuations, and thus is to be regarded as a part of the stress tensor (Zel'dovich 1968, Gunn and Tinsley 1975). The uncertainties in the tests for $\tau_{0}$ and the great dif- 
ficulty introduced by the recognition of the likely importance of dynamical evolution in the tests for $q_{0}$ remove any evidence on the question of whether there is or is not a non-zero cosmological constant. It must be stressed, however, that the three parameters as determined by the techniques outlined here give essentially independent data on the evolution and structure of the universe, and all three avenues must be pursued to a satisfactory conclusion if one is to feel at all secure about the result.

The data on $\Omega_{0}$ are with a few nagging loose ends, which can and, I am sure, will be straightened out before tests for $q_{0}$ and $\tau_{0}$ yield good answers) supportive of the conclusion that the mean density in the universe is more than an order of magnitude below the "critical" density, the closure density in the $\Lambda=0$ models. If one demands that $\Lambda$ be zero, there is only one parameter, and the conclusion is that the universe is probably open by a wide margin and will expand forever. I think, however, it behooves one to wait and see.

\section{ACKNOWLEDGMENTS}

This research was supported by a grant from the A.P.SIoan Foundation and by grants AST75-01398 and MPS74-23453 AOI from the National Science Foundation. Thanks are due also to Drs. Scott Tremaine, Jeremiah Ostriker, and Beatrice Tinsley for helpful conversations.

\section{REFERENCES}

Auer, L. and Demarque, P. 1976, Ap. J. in press.

Austin, T., Godwin, J., and Peach, J. I975, M.N.R.A.S. 171, 135. Bahcall, N. 1973, Ap. J., 180, 699.

Bautz, L. and Abell, G. 1973, Ap. J., 184, 709.

Brans, C. and Dicke, R. 1961, Phys. Rev., 124, 925.

Colla, G., Fanti, C., Fanti, R., Gioia, I., Lara, C., and Ulrich, M. 1975, Astron. and Ap., 38, 209.

de Vaucouleurs, G. 1976, Ap. J., 205, 13.

Fal1, S. M. 1976, M.N.R.A.S., I70, 181.

Geller, M. and Peebles, P.J.E. 1974, Ap. J., 184, 329.

Gott, J.R., Gunn, J.E., Schramm, D., and Tinsley, B. 1974 (GGST) Ap. J., 194,543 .

Gott, J.R. and Rees, M. 1975, Astron. and Ap., 45, 365.

Gott, J.R. and Turner, E. 1976, Ap. J., in press.

Gott, J.R. and Gunn, J.E. 1976, in preparation.

Gunn, J.E. 1974, Comments on Ap. and Space Science, 6, 7.

1976, in preparation.

Gunn, J.E. and Oke, J.B. 1974 (GO), Ap. J., 195, 255.

Gunn, J.E. and Tinsley, B.M. 1975, Nature, 257, 454. 
Gunn, J.E. and Tinsley, B.M. 1976, in press.

Hainebach, K. and Schramm, D. 1976, Ap. J., in press.

Hickson, P. 1976, unpublished thesis, California Institute of Technology.

Humason, M., Mayall, N., and Sandage, A. 1956, A. J., 61, 97.

Iben, I. 1967, Ann. Rev. Ay. and Ap., 5, 571.

Jaffe, W. and Perola, G. 1976, Astron. and Ap., 46, 275.

Ostriker, J., Peebles, P.J.E., and Yahil, A. 1974, Ap. J. (Letters), 193, L1.

Ostriker, J. and Tremaine, S. 1975, Ap. J. (Letters), 202 , Ll13.

Peebles, P.J.E. 1974, Ap. J. (Letters), 189, L51.

- 1975, Ap. J., 196, 647.

- $1976 \mathrm{a}, \frac{\text { Ap. J. }}{\mathrm{Ap}} 205,318$.

Petrosian $\mathrm{V}$ 1976b, Ap. J. (Letters), 205, L109.

Rubin, V., Ford, W.K., Thonnard, N., Roberts, M., and Graham, J. 1976, preprint.

Sandage, A.R., Tammann, G., and Hardy, E. 1972, Ap. J., 172, 253.

Sandage, A. R. and Tammann, G. 1976, Ap. J., 196, 313.

Sandage, A. R. 1976, Ap. J., 205, 6 .

Sandage, A. R., Kristian, J., and Westphal, J. 1976a, Ap. J., 265,688 .

Sandage, A. R., Kristian, J., and Westphal, J. 1976b, private communication.

Schechter, P. 1976, Ap. J., 203, 297.

Scott, E. L. 1957, A. J., 62, 248.

Segal, I. 1972, Astron. and Ap., 18, 143.

Silk, J. 1974, Ap. J., 193, 525.

Tinsley, B. M. 1975, Ap. J., 198, 145.

Tinsley, B. M. and Gunn, J. E. 1976a, Ap. J., 203, 52 .

Turner, E. 1976, Ap. J., in press.

Zel'dovich, Y. B. 1968, Astron. and Ap., 18, 143.

Zwicky, F. 1957, Morphological Astronomy, (Berlin: Springer Verlag), p. 138. 


\section{DISCUSSION}

E. SOLHEIM: In addition to the tests described by Gunn it should be mentioned that a truly global test is possible in some of the models discussed. If we have a closed model with a particle horizon $\omega_{p h}>\pi$, we

Fig. 1. - Model diagram for locating Friedman models where ghost images are possible. Models with redshift at the pole, $z_{p}=1,2,4$ and $\infty$, are shown. $\Omega_{c}$ is the critical density needed to get an ever expanding model. Models between the curves $\Omega_{c}$ and $Z_{p}=\infty$ may have ghost images.

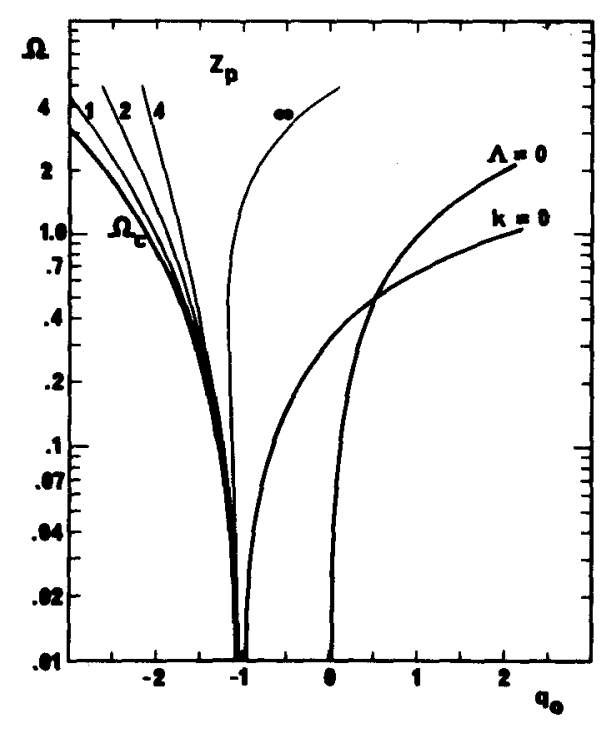

may observe the light from the same source in two diametrically opposite directions on the sky. If we can observe the redshift of both sources - this will give us a good model determination. In figure 1 is shown the part of the model diagram where this is possible.

The way to search for possible opposite images, or ghost images, is to do statistics on near opposite positions of radiosources, radiosources opposite quasars etc. It is expected that the ghost image is some angular distance away from the exact opposite direction due to transverse velocities and inhomogeneities. Figure 2 shows results of 
Fig. 2. - Number of radiosources within an angle $\Delta \theta$ of the opposite position of a quasar. Expected number is shown as a broken curve.

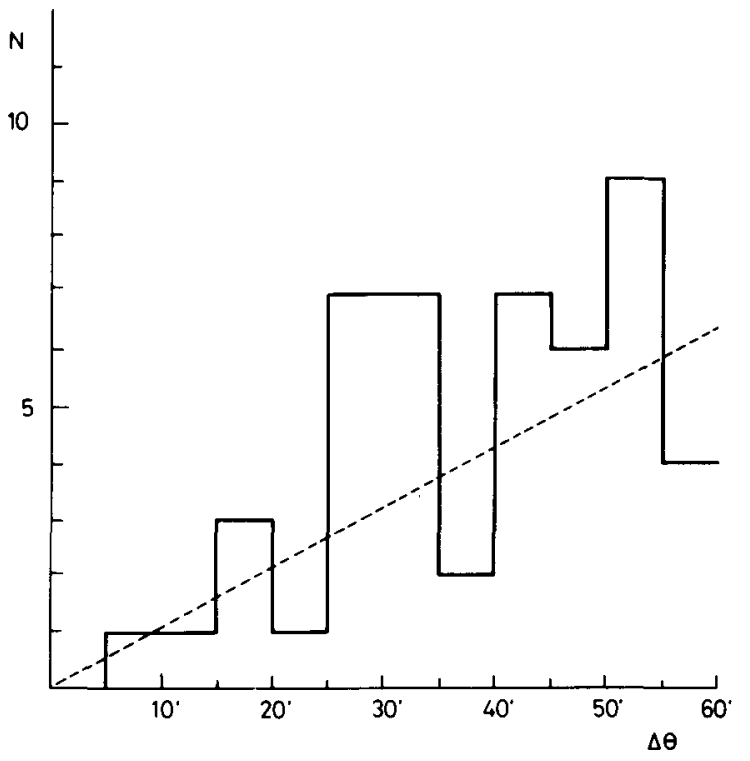

such a search among radiosources opposite quasars (Nature 1968, 219, 45). No conclusive evidence for existence of ghost images is presented so far.

J. HEIDMANN: About the age of the universe, I would like to point out, from a practical point of view, that with Campusano and Nieto (Astron. Astrophys. 1975, 4l, 229) we calculated a set of curves giving $\mathrm{H}_{O} \mathrm{t}_{\mathrm{O}}$ as a function of $\lambda_{0}$ and $\Omega_{0}$ in the ranges of interest.

Also I would like to point out that, from a study of pairs of Markarian galaxies with Kalloghlian (Astrofizica 1973, 9, 71), we obtained indication that some galaxies may be of recent origin; so in case this is confirmed, there is no reason to assume that our Galaxy was formed right after the big bang. Thus, the age of the universe could be anything larger than the 1l-18 billion years attributed to our Galaxy. J.E. GUNN: The relevant ages in the Galaxy are those of the globular. clusters: I think that the classical work of Eggen, Lynden-Bell, and Sandage showed quite convincingly that the globular clusters had to form within a few times $10^{8}$ years following the Big Bang. 
K.I. KELLERMANN: If in the future the observational situation is greatly improved with the use of the Large Space Telescope (LST), do I understand correctly that it will still be difficult to make any real progress in this conventional approach to determine $q_{0}$.

J.E. GUNN: Yes I think the difficulties now are mostly theoretical. The ST will of course be able to provide vastly better data on such things as diameters and surface brightnesses which will give much better independent handles on the evolution than we have, but as of now, there are no models to fit.

J.C. PECKER: There is still an enormous gap between the "beautiful theories" and the "horrid facts"! But, playing the "cosmological game" leads to the following comment: many more than 3 parameters are certainly necessary! For example, how do you take hierarchical universes into the reference frame of GR? Is it not true that hierarchy allows Einstein's static model to become stable? What do you feel about it?

J.E. GUNN: Hierarchical models, unless the hierarchy scale is enormous compared to the present particle horizon, are effectively ruled out, I think, by the isotropy of the 3 degree background. There is as far as I know, no real difficulty with incorporating such models in GR, but there exists no real $(\bar{\rho}=0)$ hierarchical model corresponding to the static Einstein model except ordinary Minkowski space.

J.V. NARLIKAR: I have one comment and one question. The comment is: if you take into account the possible effects of intergalactic extinction it leads to an upward revision of $q_{0}$. However, I think that near the plate limit the scott effect will become much more important and hence $q_{0}$ may have to be revised down again.

The question is: Ignoring the microwave background for the present, does your assessment of $q_{0}$ rule out the steady state model? Or are the error bars large enough to admit $q_{0}=-1$ even without the luminosity evolution of galaxies.

J.E. GUNN: It is certainly true that intergalactic extinction will lower the apparent value of $q_{0}$; I do not quite understand how this would be affected by the Scott effect. 
Our results for $q_{0}$ are only one sigma away from the steady state without evolution though I do believe that $\Omega_{0}=1$ is ruled out by the $\Omega$ tests I described. The two "physical" theories suggest that $\Omega=1$ or 2 in the steady state, but I am sure that a theory could be made consistent with the steady state hypothesis with any value of $\Omega$.

H.E. SMITH: A brief comment about the use of radio galaxies in the Hubble diagram. The recent work of Perola, Fanti and their coworkers has shown that for the upper end of the radio luminosity function there is no detectable dependence of optical luminosity on radio power. Thus, with the ability of identifying radio galaxies to much fainter limits than first ranked cluster galaxies I think they should be considered similarly useful for cosmology via the Hubble diagram, especially considering our lack of understanding of the selection effects operating on first ranked cluster galaxies.

J.E. GUNN: I think the relevant question for this recent work is whether the statistics really support no correlation at the bright end. Cosmological effects confuse this issue, of course, but the real question is whether with the small sample one can show that the slope really suddenly vanishes at the optical luminosity of the bright cluster galaxies. I find this exceedingly unlikely.

T. JAAKKOLA: It is worthwhile to emphasize the implications of the obtained low value of $q_{0}$ to our scientific world picture. According to the usual approach we would have "in the beginning" a unique, physically unconceivable event, big-bang. Since then, on the low $q_{0}$, the "dynamical" (?) evolution of the Universe would have gone on independent of the existing matter. Where then is the dynamics of this story? The "open Universe" of the present-day cosmology resembles much the Ptolemaic cosmology in which stars moved around fixed to rigid shells of the sky, there in a circular way, here galaxies in a steady radial flow, in both cases with no notion about the forces in action. However, some special theory of the Universe may be a bubble, the Universe is not!

J.E. GUNN: The low $-\Omega$ models are not "matter dominated" in the sense that the gravitation of the matter in the universe is important dynamically now, but it was early in the history of the universe, since $\Omega$ tends 
to unity in all these models as one approaches the singularity from above.

I.E. SEGAL: Dr. Gunn has chosen to devote all of his attention to the expanding-universe model. It seems appropriate therefore to indicate briefly the existence of an alternative which is physically simpler and more unique, provides a more complete theory of redshift-related questions, and fits the observational data overall far better then do Friedmann models - although it has no free parameters such as $q_{0}$ and $\wedge$, in its observable relations. This is the chronometric cosmology, which implies the frequency - independent redshift - distance relation $\mathrm{z}=$ $=\tan ^{2}(r / 2)$, where the distance $r$ is measured in units of the radius of the universe. The deductions from this law, the magnitude - redshift relation $m=2.5 \log [z /(z+1)]$ (for spectral index 1 ), etc. - fit all published data on galaxies, quasars, and radio sources quite acceptably, as regards the $(m, z),(\theta, z), N(<m), N(<z), \log N-\log s$, and similar relations; and fits the data for large or statistically well defined samples remarkably well. At the same time, the chronometric cosmology predicts a Planck law for the cosmic background radiation, and a median age for objects in the universe similar to the apparently observed ones; and eliminates quite directly many apparent anomalies based on the expansion theory, including:(a) The extraordinary energy output of quasars; (b) apparent superluminal velocities of $3 C 120$ and certain quasars; (c) the near cutoff in quasar number at redshifts $>3$; (d) The RubinFord anomaly (e) The apparently excessive dispersion in internal cluster redshifts treated by Holmberg; (f) the gap in intrinsic luminosity between the otherwise closely comparable Markarian-Seyfert galaxies and quasars; ( $g$ ) apparent variations in $\mathrm{H}$ with distance.

Physically the theory is based on general and intuitive notions of symmetry and causality, similar to those involved in particle physics. It differs from Friedmann cosmology in being unique, and involving no hypotheses as to extraordinary dynamical developments at distances or times too remote to be directly observable.

The only possibly equivocal fit to observed data is that for Sandage's sample of brightest cluster galaxies, in which case the theory fits the $(m, z)$ relation well enough to reduce the dispersion in apparent 
magnitude by a factor $\sim 2$; the expansion theory however typically does not reduce this dispersion at all, for other types of samples, and sometimes materially increases it, so this seems not a bad fit. On the other hand, the expansion theory formally reduces the dispersion by much more, which may give pause; but this is with neglect of evolution, which is important in the expansion theory. More importantly, the sample seems to lack an intrinsic criterion for inclusion of its members; involves the statistically generally ambiguous concept of 'cluster'; moreover, employs certain clusters defined by magnitude limits, whose physical (i.e. metric) implications are quite z-dependent; employs apertures appropriate to a Friedmann model, which are quite different from those for the chronometric theory; is subject to possible difficulties associated with attempted exclusion of $\mathrm{cD}$ galaxies; also the difficulty of subtracting the cluster background luminosity; and apparent internal inconsistencies in resulting estimates of the intrinsic size of the galaxies. 